\title{
UNIQUENESS THEOREM FOR THE BASIC NONSTATIONARY PROBLEM IN THE DYNAMICS OF AN IDEAL INCOMPRESSIBLE FLUID
}

\author{
V. I. YUdOVICH
}

\begin{abstract}
A ватваст. The initial boundary value problem is considered for the Euler equations for an incompressible fluid in a bounded domain $D \subset R^{n}$. It is known [Y1] that uniqueness holds for those flows with bounded vorticity. We present here a uniqueness theorem in some classes ( $B$-spaces) of incompressible flows with vorticity which is unbounded but belongs to any $L_{p}(D)$. The regularity of the flow is characterized by restrictions on the growth rate of the $L_{p}$-norms as $p \rightarrow \infty$. Roughly speaking, logarithmic singularities are forbidden but iterated logarithm singularities are permissible. It is notable that the uniqueness conditions for the Euler equations and for the motions of fluid particles are the same. The result is obtained by the energy method and a counterexample is constructed to demonstrate that it is impossible to weaken the restrictions still using the energy method.
\end{abstract}

\section{Introduction}

The basic initial boundary value problem for the Euler equations for the case of an incompressible ideal fluid is considered. The first theorems on existence and uniqueness were obtained by N. Gunter and L. Lichtenstein in two extensive series of articles $[\mathrm{Gu}, \mathrm{L}]$. They investigated the classical solutions (velocity is $C^{1, \lambda}$-smooth, $0<\lambda<1$ ) and the results were local in time. The global solvability for the 2 -dimensional problem was proved by $\mathrm{W}$. Wolibner $[\mathrm{W}]$ for the classical solutions; T. Kato $[\mathrm{K}]$ presented this result in modern form and included external forces.

The generalized solutions in the 2-dimensional case were introduced in [Y1] and the global existence theorem was proved for flows with vorticity in $L_{p}$ for any given $p>1$. However, the uniqueness theorem was obtained only for the class of flows with essentially bounded vorticity. This is the strongest known result on uniqueness whereas the results on existence were extended by A. Morgulis [M] (vorticity in Orlič spaces between $L_{1}$ and any $L_{p}, p>1$ ) and J.-M. Delort [D1, D2]. It is interesting to mention also

Received July 1, 1994. 
the result due to J.-Y. Chemin $[\mathrm{Ch}]$ on the smoothness of the boundary of the vortex patch for all time; there is another proof of this result in the paper by A. Bertozzi and P. Constantin $[\mathrm{BC}]$. See also the more detailed references in the excellent review by P. Gérard [Gé].

In the present paper the uniqueness theorem is extended to some classes of flows with unbounded vorticity. These classes are characterized by a restriction on the growth rate of the $L_{p}$-norm of vorticity; it must be sufficiently slow - not too much stronger than linear.

Two notable features of the present result should be mentioned. The first one is nonreliance of the uniqueness conditions of the dimension; only for definiteness do we treat the 3-dimensional case. Of course, according to [Y2], in the 2-dimensional case one can obtain the existence result for the classes considered here. But the $n$-dimensional case for $n \geqslant 3$ is unexpectedly difficult and remains uninvestigated for any class of flows with unbounded vorticity.

The second feature is the remarkable coincidence of uniqueness conditions for two seemingly quite different problems: for the Euler equations (uniqueness of velocity field) and for the ODE system for the translation of the fluid particles (uniqueness of the particle path).

\section{Generalized ideal flows}

The movement of an ideal incompressible fluid in a bounded domain $D \subset R^{n}$ with impermeable boundary $\partial D$ is governed by the Euler equations

$$
\begin{aligned}
\frac{\partial v_{i}}{\partial t}+v_{k} \frac{\partial v_{i}}{\partial x_{k}} & =-\frac{\partial P}{\partial x_{i}}+F_{i}, \\
\frac{\partial v_{i}}{\partial x_{i}} & =0
\end{aligned}
$$

with boundary condition

$$
\left.v \cdot n\right|_{\partial D}=0
$$

and initial condition

$$
\left.v\right|_{t=0}=v_{0} .
$$

Here $x=\left(x_{1}, \ldots, x_{n}\right) \in R^{n}, t$ denotes time, $v=\left(v_{1}, \ldots, v_{n}\right)$ is the velocity field, $P$ denotes pressure, $F=F(x, t)$ is a given field of external mass forces, and $n$ is the unit normal vector to $\partial D$. The field $v_{0}$ is the initial velocity field, which must be solenoidal, i.e., div $v_{0}=0$, and tangent to 
the boundary, i.e., $\left.v_{0} \cdot n\right|_{\partial D}=0$. The summation sign over $i$ in (1.2) and $k$ in (1.1), $i, k=1, \ldots, n$ is omitted by the usual rule.

The movement of a fluid particle, placed at a point $a \in D$ at $t=0$, is defined as the solution of the Cauchy problem

$$
\dot{x}=v(x, t),\left.\quad x\right|_{t=0}=a .
$$

The generalized solution of the problem (1.1)-(1.2) on the time interval $[0, \tau]$ (we let $\tau$ be positive, but that is not essential due to the reversibility of the problem (1.1)-(1.4)) is by definition the time-dependent vector field $v(x, t), x \in D, t \in[0, \tau]$ satisfying the following conditions:

i) The field $v$ is a vector function strongly continuous in $t \in[0, \tau]$ with values in the subspace $S_{2}(D)$ of $L_{2}(D)$, which is the closure of the set $M$ of $C^{\infty}$-smooth, finite, $n$-dimensional, solenoidal vector fields.

ii) The vector field $v$ has generalized derivatives $\partial v_{i} / \partial x_{k}, \partial v_{i} / \partial t$ in the cylinder $Q_{\tau}=D \times[0, \tau]$, and for any $p>1$ the $L_{p}(D)$-norms are bounded in $t$ :

$$
\operatorname{ess} \sup \left\{\|\nabla v(\cdot, t)\|_{L_{p}(D)}+\left\|\frac{\partial v(\cdot, t)}{\partial t}\right\|_{L_{p}(D)}: \quad t \in[0, \tau]\right\}<\infty .
$$

iii) For any smooth and solenoidal vector field $\Phi(x, t)$ which satisfies the conditions i) and ii), the following integral identity holds:

$$
\begin{array}{r}
\int_{D} v_{i}(x, \tau) \cdot \Phi_{i}(x, \tau) d x-\int_{D} v_{0 i}(x, 0) \cdot \Phi_{i}(x, 0) d x \\
=\int_{0}^{\tau} \int_{D}\left[v_{i}\left(\frac{\partial \Phi_{i}}{\partial t}+v_{k} \frac{\partial \Phi_{i}}{\partial x_{k}}\right)+\Phi_{i} F_{i}\right] d x d t .
\end{array}
$$

\section{Energy method}

Let $v, v^{\prime}$ be the generalized solutions of the problem (1.1)-(1.4) and their difference $v-v^{\prime}=u$. Taking the difference of the corresponding equations (1.6) and putting $\Phi=u$, one obtains the relation

$$
\int_{D} u^{2}(x, t) d x=-\int_{0}^{t} \int_{D} \varepsilon_{i k} u_{i} u_{k} d x d t .
$$

Here $\varepsilon_{i k}$ is the $i, k$-component of the tensor of rate of deformation,

$$
\varepsilon_{i k}=\frac{1}{2}\left(\frac{\partial v_{i}}{\partial x_{k}}+\frac{\partial v_{k}}{\partial x_{i}}\right) .
$$


The only known way to prove uniqueness is to use this equation. We call this procedure the energy method.

Let us introduce the notation

$$
f=u^{2}, \quad g=\sqrt{\varepsilon_{i k} \varepsilon_{i k}}, \quad L=L(t)=\int_{D}|f(x, t)| d x .
$$

The equation (2.1) implies the inequality

$$
L(t) \leqslant \int_{0}^{t} \int_{D} g f d x d s
$$

Uniqueness follows immediately from (2.1) if the field $v$ has bounded derivatives $\partial v_{i} / \partial x_{k}$ in the cylinder $Q_{\tau}$ or, more precisely, the estimate $|g(x, t)| \leqslant$ $b(t)$ for the rate of deformation is fulfilled for any $t \in[0, \tau]$, where $b(t)$ is some nonnegative and integrable function on $[0, \tau]$. Then by Gronwall's inequality one has as a consequence of (2.2) the inequality

$$
L(t) \leqslant L(0) \exp \int_{0}^{t} b(s) d s=0 .
$$

Thus $L(t)=0$ for $t \in[0, \tau]$, and so $u=0$.

The more accurate estimates in [Y1] permitted us to obtain uniqueness in the class of velocity fields with essentially bounded vorticity (in the 2dimensional case, but this restriction of dimension is not of importance). This is the strongest result on uniqueness known until now. We will further expand the uniqueness theorem to some classes of velocity fields with unbounded vorticities.

\section{Some integral inequalities}

It is sufficient in this section to assume that $D \subset R^{m}$ is any measurable set. Let $f, g$ be real functions on $D$, and

$$
f \in L_{1}(D) \bigcap L_{\infty}(D), \quad g \in \bigcap_{p \geqslant p_{0}} L_{p}(D)
$$

for some known $p_{0} \geqslant 1$ and assume that the following estimates are fulfilled:

$$
\|f\|_{L_{1}(D)} \leqslant L, \quad\|f\|_{L_{\infty}(D)} \leqslant M, \quad\|g\|_{L_{p}(D)} \leqslant K \phi(p),
$$


with some known positive constants $L, M>0$ and nonnegative constant $K \geqslant 0$. The given function $\phi(p)$ is strictly positive on $\left[p_{0}, \infty\right)$ and the function $\psi=T \phi$ on the positive ray $R_{+}=\{a: a \geqslant 0\}$ is defined by the equalities

$$
\begin{gathered}
\psi(a)=\inf \left\{a^{\varepsilon} \phi(1 / \varepsilon): 0<\varepsilon \leqslant 1 / p_{0}\right\}, \quad \text { for } a \geqslant 1 \\
\psi(a)=\inf \left\{\phi(1 / \varepsilon): 0<\varepsilon \leqslant 1 / p_{0}\right\}, \quad \text { for } a<1 .
\end{gathered}
$$

Lemma 3.1. The following inequality holds:

$$
\left|\int_{D} f(x) g(x) d x\right| \leqslant K L \psi(M / L) .
$$

Proof. For any $\varepsilon \in\left(0,1 / p_{0}\right]$ one has

$$
\left|\int_{D} f(x) g(x) d x\right| \leqslant M^{\varepsilon} \int_{D}|f(x)|^{1-\varepsilon}|g(x)| d x .
$$

An application of the Hölder inequality with exponents $1 /(1-\varepsilon), 1 / \varepsilon$ gives the inequality

$$
\left|\int_{D} f(x) g(x) d x\right| \leqslant K L(M / L)^{\varepsilon} \phi(1 / \varepsilon),
$$

from which (3.3) follows immediately.

Let us say that two positive functions on $\left[p_{0}, \infty\right)$ are equivalent at infinity and write $\phi_{1} \sim \phi_{2}$ if $\phi_{1}(p) / \phi_{2}(p) \rightarrow 1$ as $p \rightarrow+\infty$. We will call the corresponding equivalency class the germ at infinity.

Lemma 3.2. The map $T$ acts naturally on the germs at infinity: if $\phi_{1}(p)$ and $\phi_{2}(p)$ are the strictly positive functions, and $\phi_{1} \sim \phi_{2}$, then $T \phi_{1} \sim T \phi_{2}$.

Proof. By the equivalency condition for any $\delta>0$, there exists $q=q(\delta)$ such that for any $p>q$,

$$
(1+\delta)^{-1} \leqslant \phi_{1}(p) / \phi_{2}(p) \leqslant 1+\delta
$$

But for sufficiently large $a$ and $i=1,2$,

$$
\left(T \phi_{i}\right)(a)=\psi_{i}(a)=\inf \left\{a^{\varepsilon} \phi_{i}(1 / \varepsilon): 0<\varepsilon<1 / q\right\}
$$


To prove this, it is sufficient to note that

$$
\inf \left\{a^{\varepsilon} \phi_{i}(1 / \varepsilon): 1 / q<\varepsilon<1 / p_{0}\right\} \geqslant B a^{1 / q},
$$

where the constant $B$ is defined by

$$
B=\min _{i=1,2} \inf \left\{\phi_{i}(1 / \varepsilon): 1 / q<\varepsilon<1 / p_{0}\right\}>0 .
$$

Since the right-hand side of (3.5) is not more than $a^{1 / 2 q} \phi_{i}(2 q)$, the equality (3.5) holds at least for $a>\max \left[\phi_{i}(2 q) / B\right]^{2 q}, i=1,2$. For such $a$ one deduces from (3.4) and (3.5) that

$$
(1+\delta)^{-1} \leqslant \psi_{1}(a) / \psi_{2}(a) \leqslant 1+\delta
$$

Thus $\psi_{1}(a) / \psi_{2}(a) \rightarrow 1$ when $a \rightarrow+\infty$.

Example 3.1. Let $\phi_{0}(p)=p$. Then $\psi_{0}=T \phi_{0}$ is given by

$$
\psi_{0}(a)= \begin{cases}T\left(\phi_{0}\right)(a) e \ln a, & a>\exp p_{0} \\ T\left(\phi_{0}\right)(a) p_{0} a^{1 / p_{0}}, & 1 \leqslant a \leqslant p_{0} \\ T\left(\phi_{0}\right)(a) p_{0}, & 0 \leqslant a \leqslant 1 .\end{cases}
$$

Of course, the first line only is of real interest.

According to Lemma 3.1 we have the inequality

$$
\left|\int_{D} f(x) g(x) d x\right| \leqslant e K L \ln (M / L)
$$

for sufficiently large $M / L$ (specifically, for $M / L>\exp p_{0}$ ).

We will now generalize this example. Let us put for any natural $m$

$$
\theta_{m}(p)=\ln p \cdot \ln \ln p \ldots \ln _{m} p
$$

where $\ln _{m}$ means the $m$-th iteration of logarithm; $p$ is positive and sufficiently large $\left(p>\exp _{m-1} 1\right)$; and $\exp _{0}=\mathrm{id}$ by definition. Also by definition $\theta_{o}(p)=1$. The functions $\phi_{1}, \phi_{2}, \ldots$ are defined recursively:

$$
\phi_{0}(p)=p ; \quad \phi_{m}(p)=\phi_{0}(p) \theta_{m}(p), \quad m=1,2, \ldots
$$


Lemma 3.3. Let the inequalities (3.1)-(3.3) be fulfilled with $\phi=\phi_{m-1}$ for some natural number $m$. Then for sufficiently large ratio $M / L(M / L>$ $\left.\exp _{m} 1\right)$ the following inequality holds:

$$
\left|\int_{D} f(x) g(x) d x\right| \leqslant e K L \theta_{m}(M / L) .
$$

Proof. Let us denote $T \phi_{m-1}=\psi_{m}$ and $\varepsilon_{*}=1 / \ln a$. For large $a(a \geqslant$ $\left.\exp p_{0}\right)$,

$$
\psi_{m}(a) \leqslant a^{\varepsilon_{*}} \phi_{m-1}\left(1 / \varepsilon_{*}\right)=e \theta_{m}(a) .
$$

Inequality (3.9) follows from Lemma 3.1 and inequality (3.10).

Under the conditions of Lemma 3.3, it is easy to prove that $\psi_{m} \sim e \theta_{m}$. It is sufficient to note that the minimizing value of $\varepsilon$ for the function $a^{\varepsilon} \phi_{m-1}(1 / \varepsilon)$ is equivalent asymptotically to just $\varepsilon_{*}=1 / \ln a$.

The following examples help us to understand the nature of the requirement imposed on the function by the condition (3.3).

Example 3.2. Set $B_{1}=\left\{x: x \in R^{n},|x| \leqslant 1\right\}$, the unit ball in $R^{n}$. Let the function $g_{1}$ on $B_{1}$ be defined by $g_{1}(x)=\ln r, r=|x|$. Then one has

$$
\left\|g_{1}\right\|_{L_{p}\left(B_{1}\right)}^{p}=\sigma_{n} n^{-(p+1)} \Gamma(p+1) .
$$

Here $\sigma_{n}$ is the surface of the unit sphere $\partial B_{1}$. By Stirling's formula one obtains

$$
\left\|g_{1}\right\|_{L_{p}\left(B_{1}\right)} \sim p /(e n) \quad \text { as } p \rightarrow \infty
$$

Example 3.3. Let $g_{2}(x)=\ln \ln (e / r)$ for $x \in B_{1}$. Then

$$
\left\|g_{2}\right\|_{L_{p}\left(B_{1}\right)}^{p}=\sigma(\exp n) \int_{0}^{\infty} \exp (p \ln s-n \exp s+s) d s .
$$

One can find the asymptotics of this integral by Laplace's method. As a result one has

$$
\left\|g_{2}\right\|_{L_{p}\left(B_{1}\right)} \sim \ln p \quad \text { for } p \rightarrow \infty
$$




\section{Estimate of velocity by vorticity}

In this section we will consider the classical boundary value problem of vector analysis

$$
\begin{aligned}
& \operatorname{div} \mathbf{v}=0 \\
& \operatorname{curl} \mathbf{v}=\omega, \\
&\left.\mathbf{v}_{n}\right|_{\partial D}=0 \\
& \int_{\gamma_{k}} \mathbf{v} \cdot d s=0, \quad k=1,2, \ldots, l .
\end{aligned}
$$

The unknown vector field $\mathbf{v}$ is defined on the bounded domain $D \subset R^{3}$ with $C^{2}$-smooth boundary $\partial D$. According to $(4.3) \mathbf{v}$ is tangent to $\partial D$. Here $\omega$ is a given vector field. The closed curves $\gamma_{1}, \gamma_{2}, \ldots, \gamma_{l}$ form the basis of one-dimensional singular homologies of the domain $D$. The known vector field $\omega$ must be solenoidal at least in the sense of distributions; this is a necessary condition for solvability. The existence of a solution and the estimates in Hölder and $L_{p}$-norms were the objects of much work (see $[\mathrm{F}, \mathrm{BS}, \mathrm{S}])$.

Lemma 4.1. For any $p>1$, the following estimate holds:

$$
\|\nabla \mathbf{v}\|_{L_{p}(D)} \leqslant \frac{C p^{2}}{p-1}\|\omega\|_{L_{p}(D)}
$$

with the constant $C$ depending only on $D$, and not on $p$.

Lemma 4.2. The solution of the problem (4.1)-(4.4) for any $\lambda \in(0,1)$ satisfies the inequality

$$
\left|v\left(x^{\prime}\right)-v\left(x^{\prime \prime}\right)\right| \leqslant C[\lambda(1-\lambda)]^{-(\lambda+2) / 3}\|\omega\|_{L_{p}(D)} \cdot\left|x^{\prime}-x^{\prime \prime}\right|^{\lambda}
$$

for any points $x^{\prime}, x^{\prime \prime} \in D, p=3 /(1-\lambda)$. The constant $C$ depends only on $D$, and not on $\lambda$.

The estimates (4.5)-(4.6) give the exact order of the growth for $p \rightarrow 1$ or $p \rightarrow \infty$, and $\lambda \rightarrow 0,1$ respectively. To prove the estimate (4.5) for simple domains such as the whole space $R^{3}$, the half-space or the ball, it is sufficient to apply the classical result of Calderon-Zygmund [CZ]. As for the general case the proof is based on a rather awkward technique, developed in [Y1, Y2]. It is convenient to deduce the inequality (4.6) together with (4.5), applying the standard methods of potential theory.

The estimates (4.5), (4.6) are valid also in the general case of $n$-dimensional compact manifolds with boundary, $p=n /(1-\lambda)$ and the exponent $(\lambda+n-1) / n$ instead of $(\lambda+2) / 3$ in $(4.6)$. 


\section{Uniqueness}

For any positive germ $\theta$ on the ray $R_{+}$, the class $R_{\theta}$ of vector fields on $D$ is defined by the requirement that for sufficiently large $p\left(p \geqslant p_{0}\right.$, with dependence on $p_{0}$ of the field permitted) the estimate

$$
\|\operatorname{curl} \mathbf{v}\|_{L_{p}(D)} \leqslant K \theta(p)
$$

holds with $K$ not depending on $p$. Let us denote by $K_{\theta}(\mathbf{v})$ the seminorm

$$
K_{\theta}(\mathbf{v})=\varlimsup_{p \rightarrow+\infty} \frac{1}{\theta(p)}\|\operatorname{curl} \mathbf{v}\|_{L_{p}(D)} .
$$

The function $\theta$ is called admissible if for $\psi=T(p \theta)$ the following integral diverges:

$$
\int^{+\infty} \frac{d a}{a \psi(a)}=\infty .
$$

By $R_{\theta, \tau}$ we will denote the class of time-dependent vector fields on $D$ in which the seminorm (5.2) is a measurable and essentially bounded function of $t \in[0, \tau]$.

Theorem 1. There exists not more than one solution of the problem (1.1)(1.4) on any time interval $[0, \tau], \tau>0$, in the class $R_{\theta, \tau}$ with admissible function $\theta$.

Proof. Estimating the integral (2.2) by Lemma 4.1, one has inequality

$$
L(t) \leqslant \int_{0}^{t} \beta(L(s)) d s,
$$

where the function $\beta$ and the constant $M$ are given by

$$
\beta(L)=K L \psi(M / L), \quad M=\max \left\{u^{2}(x, t): \quad(x, t) \in D \times[0, \tau]\right\} .
$$

It follows from (5.4) that $L(t) \equiv 0$ by Osgood's uniqueness theorem $[\mathrm{H}]$.

Theorem 2. For any point $a \in D$ the Cauchy problem

$$
\dot{x}=v(x, t), \quad x(0)=a
$$

has a unique solution for any vector field $\mathbf{v}$ in $R_{\theta, \tau}$ with the admissible function $\theta$.

Proof. Putting $\lambda=1-3 \varepsilon$ in (4.6) and taking the exact lower bound in $\varepsilon$ one obtains the inequality

$$
\left|v\left(x^{\prime}, t\right)-v\left(x^{\prime \prime}, t\right)\right| \leqslant C K_{1} h \psi\left(h^{-3}\right), \quad K_{1}=K \cdot 4^{-4} .
$$

This is sufficient to deduce the existence of solution by Peano's theorem and uniqueness by Osgood's theorem. 


\section{Counterexamples}

Now we will construct some counterexamples in order to show that it is impossible to diminish the regularity restrictions on the generalized solutions in Theorem 1, at least in the framework of the energy method. More general counterexamples can be constructed and we hope to include them in a more complete publication. Thus it seems that if one would like to strengthen the result of this theorem, one has to invent a new method.

Let us define the function $f(x, t)$ for $(x, t) \in B_{1} \times[0, \tau]$, where $B_{1}$ is the unit disc on $R^{2}$ and $\tau$ is positive number, by

$$
f(x, t)=\left\{\begin{array}{ll}
1, & \text { for } r \leqslant a(t), \\
0, & \text { for } r>a(t),
\end{array} \quad r=|x| .\right.
$$

Let us try to find a nonnegative function $a(t)$ such that $a(t) \rightarrow 0$ when $t \rightarrow+0$, and the equality

$$
\frac{d}{d t} \int_{D} f d x=\int_{D} g f d x
$$

holds for small positive $t$ and some function $g$. From the assumption that $g=g(r), r$ being the polar radius, it follows that

$$
\frac{d a}{d t}=\frac{1}{a} \int_{0}^{a} g(r) r d r \stackrel{\text { def }}{=} G(a) .
$$

Let $g(r)$ be a continuous function on $(0,1]$, positive and decreasing. If

$$
\int_{+0} \frac{d a}{G(a)}<\infty
$$

then the differential equation (6.3) has a nontrivial solution $a(t)$ such that $a(0)=0$. It is defined implicitly by the equation

$$
\int_{0}^{a(t)} \frac{d b}{G(b)}=t .
$$

Thus, if (6.4) holds, the equation (6.2) does not imply the equality $f=0$. Now let

$$
g(r)=[\ln (e / r)]^{\alpha+1}
$$


Then (6.4) holds only for $\alpha \geqslant 0$. On the other hand, for the function (6.6), putting $\phi(p)=p \theta(p)=\|g\|_{L_{p}\left(B_{1}\right)}, \psi=T \phi$, we have (see Example 3.2)

$$
\theta(p)=O\left(p^{\alpha}\right), \quad \psi\left(e^{z}\right)=O\left(z^{\alpha+1}\right), \quad p, z \rightarrow+\infty
$$

It follows that the condition (5.3) holds if and only if $\alpha \leqslant 0$. Thus, for the classes $R_{\theta}, \theta(p)=p^{\alpha}$ the condition (5.3) is exact.

The same results are true for the class $R_{\theta}$ with $\theta(p)=(\ln p)^{\alpha}$. For this purpose it is sufficient to note that for the functions

$$
g(r)=\ln (e / r) \cdot[\ln \ln (e / r)]^{\alpha+1}
$$

the condition (6.4) holds for $\alpha>0$ while the condition (5.3) holds if and only if $\alpha \leqslant 0$.

\section{Acknowledgement}

This work received support from Russian Foundation for Fundamental Sciences (grant \# 93-013-17337) and from NSF (grant DMS-9300752). I would like to thank Susan Friedlander, Andrey Morgulis and Misha Vishik for interesting discussions concerning this work. The author is grateful also to Julia Zhdanova for her help in preparation of this article.

\section{References}

[BC] A. Bertozzi and P. Constantin, Global Regularity for Vortex Patches, Comm. Math. Phys. 152 (1993), 19-28.

[BS] E. Bykhovsky and N. Smirnov, On orthogonal decompositions of square integrable vector functions, Tr. MIAN SSSR 59 (1960), 6-36. (Russian)

[CZ] A.P. Calderon and A. Zygmund, On existence of certain singular integrals, Acta Math. 88 (1952), 85.

[Ch] J.-Y. Chemin, Persistance de structures géométriques dans les fluides incompressibles bidimensionnels, Annales de l'Ecole Normale Supérieure 26 (1993), no. 4, $1-26$.

[D1] J.-M. Delort, Existence de nappes de tourbillon pour l'équation d'Euler sur le plan, Séminaire Equations aux dérivées partielles, 1990-1991, Ecole Polytechnique.

[D2] _ Existence de nappes de tourbillon en dimension deux, J. Amer. Math. Soc. 4 (1991), no. 3, 553-586.

[F] K. Friedrichs, Differential forms on Riemannian manifolds, Comm. Pure Appl. Math. 8 (1955), 551-590.

[Gé] P. Gérard, Résultats récents sur les fluides parfaits incompressibles bidimenionnels (d'après J.-Y. Chemin et J.-M. Delort), Seminaire Bourbaki, 44ème année (199192), no. 757, 411-444.

[Gu] N. Gunter, On the motion of fluid in the moving container, Izv. Akad. Nauk SSSR. Ser. Fiz.-Mat. 20 (1926), 1323-1348, 1503-1532; 21 (1927), 621-556, 735-756, 1139-1162; 22 (1928), 9-30. 
[H] F. Hartman, Ordinary differential equations (1964), John Wiley \& Sons, NYLondon-Sidney.

[K] T. Kato, On classical solutions of the two-dimensional non-stationary Euler equation, Arch. Rat. Mech. 27 (1968), 188-200.

[L] L. Lichtenstein, Über einige Existenzprobleme der Hydrodynamik homogener unzusammendrückbarer, reibunglosser Flüssikeiten und die Helmholtzschen Wirbelsaltze, Math. Zeit. 23 (1925), 89-154; 26 (1927), 196-323, 387-415, 725; 32 (1930), 608.

[M] A. Morgulis, On existence theorem for bidimensional nonstationary flows of ideal incompressible fluid with vorticity not summable with degree more than 1, Sibirsk. Matem. Zhurn. 33 (1992), no. 5. (Russian)

[S] V. Solonnikov, Overdetermined elliptic boundary value problems., Zapiski nauch. semin. LOMI 21 (1971), no. 5. (Russian)

[W] W. Wolibner, Un théorème sur l'existence du movement plan d'un fluide parfait, homogène, incompressible, pendant un temps infiniment long, Math. Zam. 37 (1933), 698-726.

[Y1] V. Yudovich, Nonstationary flow of an ideal incompressible liquid, Zhurn. Vych. Mat. 3 (1963), 1032-1066. (Russian)

[Y2] _ Some bounds for solutions of elliptic equations, Mat. Sb. 59 (1962), no. 101, 229-244; English transl. in Amer. Math. Soc. Transl. (2) 56 (1966).

Department of Mathematics, Rostov State Univerity, 5 Zorge, RostovON-D ON, 344104 RUSSIA

E-mail address: kraiz@rsu.rnd.su 